\title{
ДИСЛОКАЦИОННАЯ КИНЕТИКА ПРИ ПЛАСТИЧЕСКОЙ ДЕФОРМАЦИИ ДВУМЕРНЫХ ПОЛИКРИСТАЛЛОВ
}

\author{
Е.Е. Бадиян, А.Г. Тонкопряд, Е.В. Фтемов, О.В. Шеховцов \\ Харьковский национальный университет им. В.Н. Каразина, Харьков, Украина \\ E-mail: Evgeny.E.Badiyan@univer.kharkov.ua; meл. +38(057)707-53-47
}

\begin{abstract}
Дислокационно-кинетический подход применен к исследованию пластического течения плоских образцов двумерных поликристаллов чистых металлов в условиях одноосного растяжения с постоянной скоростью деформации при умеренных температурах. Сформулировано дислокационно-кинетическое уравнение, в котором учтены роль свободной поверхности плоского образца, являющейся источником и стоком дислокаций, и упрочняющее действие сквозных границ зерен в двумерном поликристалле. Для расчета кривой деформации кинетическое уравнение преобразовано с использованием закона деформационного упрочнения Тейлора и получено аналитическое решение этого уравнения. На примере плоских образцов двумерных поликристаллов чистого алюминия (99,999 ат.\%) показано, что результаты расчетов достаточно хорошо согласуются с экспериментальными данными.
\end{abstract}

\section{ВВЕДЕНИЕ}

Широкое использование различных поликристаллических твердых тел в качестве конструкционных материалов обусловливает необходимость понимания физической природы их прочности и пластичности. Как известно, границы зерен являются не только барьерами для движения дислокаций, контролирующих механические свойства поликристаллов, но и эффективными источниками и стоками дислокаций и других радиационных дефектов, обеспечивающими повышенную радиационную стойкость [1-3]. Удачным модельным объектом для исследования являются двумерные поликристаллы. Они содержат только один слой зерен и имеют сквозные «вертикальные» границы зерен, т. е. все зерна являются «поверхностными» в том смысле, что они имеют выход на свободную поверхность образца. В двумерных поликристаллах существует возможность определения кристаллографической ориентации всех зерен и кристаллогеометрических параметров их границ. Из-за отсутствия стесненности «поверхностных» зерен в «вертикальном» направлении, перпендикулярном оси растяжения, специфически проявляются ротационные $[4,5]$ и другие эффекты [6, 7], связанные с пластической деформацией. Вместе с тем двумерные поликристаллы находят самостоятельное практическое применение в качестве поликристаллических пленок, фольг и пластин, которые эксплуатируются в условиях действия механических напряжений.

Для описания пластического течения кристаллического материала может быть использован дислокационно-кинетический подход. Он основан на уравнениях, описывающих эволюцию плотности дислокаций в материале с ростом степени пластической деформации. Такой подход позволяет получить зависимость напряжения течения от среднего размера зерен, поперечного размера образца, температуры, степени и скорости деформации [8-14].

\section{1. ДИСЛОКАЦИОННО-КИНЕТИЧЕСКОЕ УРАВНЕНИЕ ДЛЯ ПЛОСКИХ ОБРАЗЦОВ ДВУМЕРНЫХ ПОЛИКРИСТАЛЛОВ}

Настоящая работа посвящена исследованию пластической деформации в условиях одноосного растяжения при умеренных температурах плоских образцов двумерных поликристаллов чистых металлов с толщиной от $\sim 50$ мкм и более и со средним размером зерен от $\sim 50$ мкм и до макроскопических размеров (нано- и микроразмерные образцы с нано- и микрозеренной структурами исследованы в работах [12-14]). Под плоскими образцами здесь понимаются образцы прямоугольного сечения, размеры которых связаны соотношением $D \ll w<l$, где $D$ - толщина образца (размер в «вертикальном» направлении), w и $l$ ширина и длина рабочей части соответственно (рис. 1). В таких образцах имеем отношение площади свободной поверхности к объему: $S_{S} / V \gg 1 \mathrm{~cm}^{-1}$

Кинетическое уравнение описывает эволюцию средней плотности дислокаций $\rho$ в материале с ростом сдвиговой деформации $\gamma$. Оно должно содержать произведение $\rho(d \rho / d \gamma)$ и слагаемые, описывающие процессы накопления дислокаций в материале и уменьшения их плотности. Запишем дислокационно-кинетическое уравнение для плоских образцов двумерных поликристаллов, используя данные работ [8-14]. При этом должна быть учтена специфика исследуемых образцов. Она состоит в том, что в таких объектах большую роль играет свободная поверхность образца, которая является источником и стоком дислокаций. Кроме того, все границы зерен являются сквозными, что обусловливает особенности связанного с ними деформационного упрочнения.

Накопление дислокаций в трехмерном поликристалле, толщина $D$ которого значительно превосходит средний размер зерен $d$, вследствие наличия границ зерен описывается членом $(\beta / b d) \rho$, где $b$-вектор Бюргерса; $\quad \beta$-коэффициент, определяющий интенсивность накопления дислокаций в зернах в результате ограничения длины свободного пробега дислокаций размером зерна $d$. 


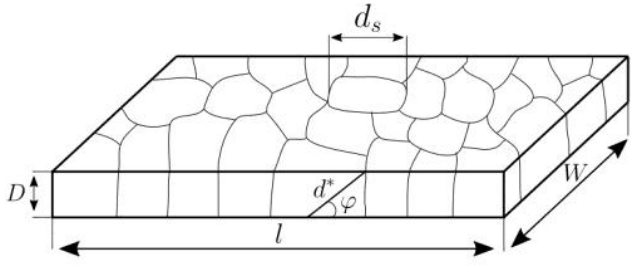

Рис. 1. Схема плоского образияа двумерного поликристалла

Коэффициент $\beta$ выражает относительную долю зерен, заключенных в объеме и не имеющих выхода на свободную поверхность образца. Однако в двумерном поликристалле все зерна являются «поверхностными», поэтому $\beta=0$. Здесь упрочняющее действие оказывают сквозные «вертикальные» границы зерен, и нам необходимо его учесть.

Согласно [7] «вертикальные» границы «поверхностных» зерен создают препятствия для движения дислокаций только в тех областях зерен, которые примыкают к этим границам. Таким образом, в «поверхностном» зерне, объем которого равен $V$, имеется его часть с объемом $V^{*}$, в котором дислокации испытывают затруднение для движения, встречаясь с «вертикальными» границами зерен, в то время как в остальной части зерна объемом $V-V^{*}$ движущиеся дислокации таких препятствий не имеют. Относительную часть зерна, которая примыкает к «вертикальным» границам, определяет величина $p^{*}=V^{*} / V$. Тогда по аналогии с $\beta / b d$ можем записать выражение $p^{*} / b d^{*}$, которое характеризует упрочняющее действие «вертикальных» границ зерен в двумерном поликристалле. Длина свободного пробега $d^{*}$ дислокаций в «поверхностном» зерне до встречи с вертикальной границей (см. рис. 1) нуждается в определении.

Если принять, что «поверхностные» зерна имеют форму квадрата со стороной $d_{s}$ на свободной «горизонтальной» поверхности и размер $d_{t}=D$ в поперечном («вертикальном») направлении, то в случае $d_{s} \leq d_{t}$ имеем дело с зеренной структурой, которая при $d_{s} \ll d_{t}$ является «игольчатой». Для нее $p^{*}=1$ и $d^{*}=d_{s} / \cos \varphi$, где $\varphi-$ угол между плоскостью скольжения и осью растяжения (вдоль этой оси измеряется длина $l$ рабочей части образца). Значит, в этом случае $p^{*} / b d^{*}=\cos \varphi / b d_{s}$. Для зеренной структуры с параметрами $d_{s}>d_{t}$, которая при $d_{s} \gg d_{t}$ является «блинной», получается $p^{*}=\left(d_{t} / d_{s}\right) / \operatorname{tg} \varphi$ и $d^{*}=d_{t} / \sin \varphi$, но выражение $p^{*} / b d^{*}=\cos \varphi / b d_{s}$ остается без изменений. Таким образом, в дислокационно-кинетическом уравнении зернограничное упрочнение в двумерном поликристалле описывается членом $\left(p^{*} / b d^{*}\right) \rho=$ $\left(\cos \varphi / b d_{s}\right) \rho$.

Кроме рассмотренного выше зернограничного упрочнения, вклады в накопление дислокаций дают также работа поверхностных дислокационных источников с плотностью $n_{S}$ и размножение дислокаций посредством механизма двойного поперечного скольжения винтовых дислокаций на дислокациях леса. В кинетическом уравнении они описываются слагаемыми $\left(\frac{S_{S}}{V}\right)\left(\frac{n_{S}}{b}\right)$ и $k_{f} \rho^{3 / 2}$ соответственно, где $k_{f}$-коэффициент, определяющий интенсивность размножения дислокаций на дислокациях леса $\left(k_{f} \approx 10^{-2} / b\right)$. Следует отметить, что механизм размножения дислокаций на дислокациях леса характерен для так называемых обычных поликристаллических металлов с размерами зерен от 10 мкм и более, но не работает в нанокристаллических материалах [12, 14]. Для образцов прямоугольного сечения отношение площади свободной поверхности к объему определяется выражением $S_{S} / V=2(1 / D+$ $1 / w)$, которое для плоских образцов $(D \ll w)$ преобразуется к виду $S_{S} / V=2 / D$ [13].

Выпадение дислокаций из процесса размножения вследствие их выхода из образца на его поверхность приводит к уменьшению средней плотности $\rho$. В кинетическом уравнении такой процесс учитывает член $-(\sin \varphi / b D) \rho$. Кроме того, плотность дислокаций в материале уменьшается из-за аннигиляции винтовых участков дислокационных петель, что учитывает слагаемое $-k_{a} \rho^{2}$, где $k_{a}-$ коэффициент аннигиляции винтовых дислокаций.

В итоге для плоских образцов двумерных поликристаллов чистых металлов с толщиной от $\sim 50$ мкм и более и со средним размером зерен от $\sim 50$ мкм и до макроскопических размеров в условиях одноосного растяжения с постоянной скоростью деформации $\dot{\varepsilon}$ при умеренных температурах (в отсутствие диффузионных механизмов аннигиляции дислокаций) дислокационнокинетическое уравнение может быть записано в виде

$$
\begin{gathered}
\rho(d \rho / d \gamma)=\left(\cos \varphi / b d_{s}\right) \rho+(2 / D)\left(n_{S} / b\right)+k_{f} \rho^{3 / 2}- \\
(\sin \varphi / b D) \rho-k_{a} \rho^{2} .
\end{gathered}
$$

\section{2. ПЛАСТИЧЕСКОЕ ТЕЧЕНИЕ ПЛОСКИХ ОБРАЗЦОВ ДВУМЕРНЫХ ПОЛИКРИСТАЛЛОВ}

Зависимость напряжения $\sigma$ от степени пластической деформации $\varepsilon$ характеризует пластическое течение материала. Для получения кривых деформации $\sigma(\varepsilon)$ в случае одноосного растяжения плоских образцов двумерных поликристаллов преобразуем кинетическое уравнение (1) подобно тому, как это сделано в работах [8, 10, 14], воспользовавшись выражениями $\gamma=m \varepsilon, \sigma=m \tau$, где $m-$ ориентационный фактор; $\tau$-напряжение течения, которое определяется взаимодействием дислокаций друг с другом в соответствии с соотношением Тейлора [15]

$$
\tau=\alpha \mu b \rho^{1 / 2},
$$

в котором $\alpha$-постоянная взаимодействия дислокаций друг с другом; $\mu$ - модуль сдвига. Выполнив преобразования, получим

$$
\sigma^{3}(d \sigma / d \varepsilon)=-m k_{a}\left(\sigma^{4}+a_{1} \sigma^{3}+a_{2} \sigma^{2}+a_{3}\right) / 2,
$$

где

$$
\begin{gathered}
a_{1}=-m \alpha \mu b k_{f} / k_{a} \\
a_{2}=-\left(\cos \varphi / b d_{s}-\sin \varphi / b D\right)(m \alpha \mu b)^{2} / k_{a}, \\
a_{3}=-(2 / D)\left(n_{S} / b\right)(m \alpha \mu b)^{4} / k_{a} .
\end{gathered}
$$


Интегрируя (3), получаем зависимость деформирующего напряжения $\sigma$ от степени пластической деформации $\varepsilon$ в неявном виде:

$$
\begin{gathered}
-\left(2 / m k_{a}\right)\left(A_{1} \ln \left|\sigma-\sigma_{1}\right|+A_{2} \ln \left|\sigma-\sigma_{2}\right|\right. \\
+\left(A_{3} / 2\right) \ln \left|\sigma^{2}+\xi_{1} \sigma+\xi_{2}\right|+ \\
+\left(\left(-\xi_{1} A_{3} / 2+A_{4}\right) / \sqrt{\xi_{2}-\left(\xi_{1} / 2\right)^{2}}\right) \operatorname{arctg}((\sigma+ \\
\left.\left.\left.\xi_{1} / 2\right) / \sqrt{\xi_{2}-\left(\xi_{1} / 2\right)^{2}}\right)\right)+C=\varepsilon .
\end{gathered}
$$

Постоянная интегрирования $C$ определяется из условия $\sigma(0)=0$. Параметры в (4) имеют вид:

$$
\begin{gathered}
\sigma_{1,2}= \\
\left.\sqrt{\left(\frac{1}{2}\right)\left(\sqrt{\frac{a_{1}^{2}}{4}-a_{2}+y_{1}}-\frac{a_{1}}{2} \pm\right.}+\sqrt{\left.\frac{a_{1}^{2}}{4}-a_{2}+y_{1}\right)^{2}-4\left(\frac{y_{1}}{2}-\sqrt{\frac{y_{1}^{2}}{4}-a_{3}}\right)}\right),
\end{gathered}
$$

где

$$
\begin{gathered}
y_{1}=\left(-q / 2+\sqrt{(p / 3)^{3}+(q / 2)^{2}}\right)^{1 / 3}+(-q / 2- \\
\left.\sqrt{(p / 3)^{3}+(q / 2)^{2}}\right)^{1 / 3}+a_{2} / 3, \\
p=-a_{2}^{2} / 3-4 a_{3}, \\
q=-2\left(a_{2} / 3\right)^{3}+8 a_{2} a_{3} / 3-a_{1}^{2} a_{3} .
\end{gathered}
$$

Величины $A_{1}, A_{2}, A_{3}, A_{4}$ определяются как решение системы уравнений

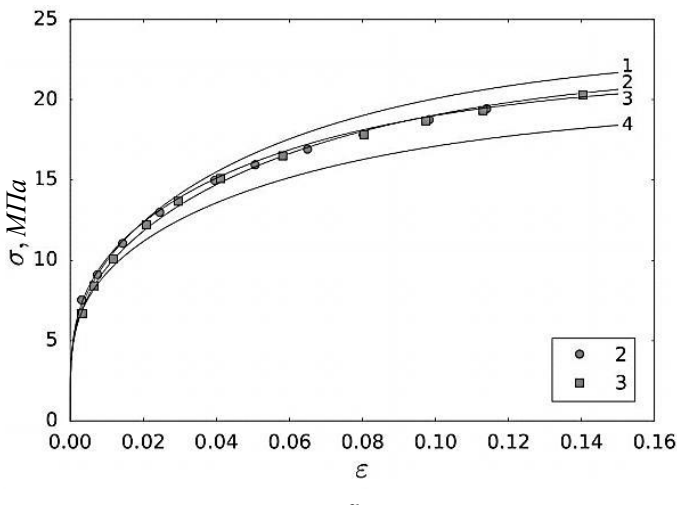

$a$

$$
\begin{gathered}
A_{1}+A_{2}+A_{3}=1 ; \\
A_{1}\left(\xi_{1}-\sigma_{2}\right)+A_{2}\left(\xi_{1}-\sigma_{1}\right)-A_{3}\left(\sigma_{1}+\sigma_{2}\right)+A_{4}=0 ; \\
A_{1}\left(\xi_{2}-\xi_{1} \sigma_{2}\right)+A_{2}\left(\xi_{2}-\xi_{1} \sigma_{1}\right)+A_{3} \sigma_{1} \sigma_{2}- \\
A_{4}\left(\sigma_{1}+\sigma_{2}\right)=0 ; \\
-A_{1} \xi_{2} \sigma_{2}-A_{2} \xi_{2} \sigma_{1}+A_{4} \sigma_{1} \sigma_{2}=0,
\end{gathered}
$$

в которой

$$
\begin{gathered}
\xi_{1}=a_{1} / 2+\sqrt{\left(a_{1} / 2\right)^{2}-a_{2}+y_{1}}, \xi_{2}=y_{1} / 2+ \\
\sqrt{\left(y_{1} / 2\right)^{2}-a_{3}} .
\end{gathered}
$$

Далее в качестве примера приводятся кривые деформации $\sigma(\varepsilon)$ в случае одноосного растяжения плоских образцов двумерных поликристаллов чистого алюминия $(99,999$ ат.\%). На рис. 2 линиями показаны зависимости $\sigma(\varepsilon)$, которые были рассчитаны в соответствии с (4) и «сшиты» с линейным участком, соответствующим упругой деформации, при значениях напряжения и деформации соответственно $0,07 \mathrm{MПа} \mathrm{и}$ $10^{-6}$ согласно данным [16]. Значения параметров, которые использовались при расчетах указанных кривых деформации по формуле (4), подобраны в соответствии с данными работ $[7,12,13,17]$ и представлены в таблице. Экспериментальные данные взяты из работы [7] и показаны на рис. 2 точками.

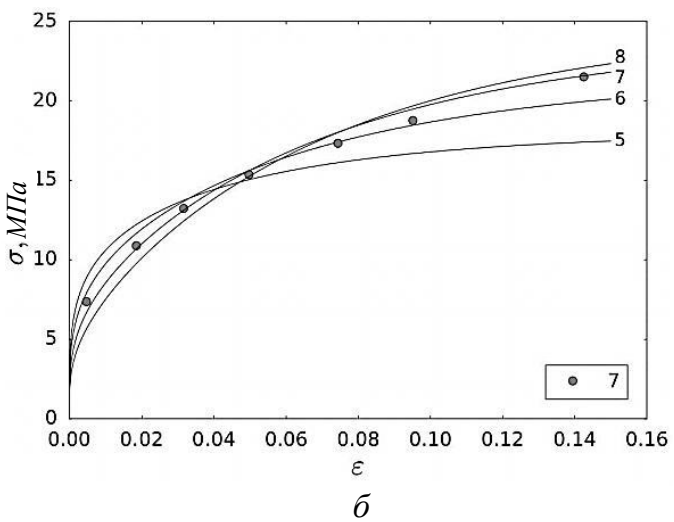

Рис. 2. Кривые деформации плоских образиов двумерных поликристаллов чистого Al (99,999 ат.\%) толщиной $D=95$ мкм с различным средним размером зерен $d_{s}$ :

$$
1 \text {-120, } 2 \text { - 183, } 3 \text {-205, 4-1000 мкм (a); }
$$

со средним размером зерен $d_{s}=379$ мкм и различной толщиной D: 5 - 50, 6-100, 7- 266, 8 - 700 мкм (б)

Значения параметров плоских образцов двумерных поликристаллов чистого $\mathrm{Al}$ (99,999 ат.\%), использованных при расчетах кривых деформации на рис. 2 в соответствии с данными $[7,12,13,17]$

\begin{tabular}{|c|c|c|c|c|c|c|c|c|c|c|}
\hline $\begin{array}{c}\text { Номер } \\
\text { кривой }\end{array}$ & $D$, мкм & $w$, мм & $d_{s}$, мкм & $m$ & $\varphi$ & $\mu$, ГПа & $b$, нм & $\alpha$ & $k_{a}$ & $n_{S}$, мкм $^{-2}$ \\
\hline 1 & 95 & 4 & 120 & 2,60 & $\pi / 4$ & 27 & 0,286 & 0,32 & 9,7 & 1,00 \\
\hline 2 & 95 & 4 & 183 & 2,65 & $\pi / 4$ & 27 & 0,286 & 0,32 & 9,7 & 0,85 \\
\hline 3 & 97 & 4 & 205 & 2,52 & $\pi / 4$ & 27 & 0,286 & 0,32 & 9,7 & 1,50 \\
\hline 4 & 95 & 4 & 1000 & 2,60 & $\pi / 4$ & 27 & 0,286 & 0,32 & 9,7 & 1,00 \\
\hline 5 & 50 & 4 & 379 & 2,69 & $\pi / 4$ & 27 & 0,286 & 0,32 & 9,7 & 1,00 \\
\hline 6 & 100 & 4 & 379 & 2,69 & $\pi / 4$ & 27 & 0,286 & 0,32 & 9,7 & 1,00 \\
\hline 7 & 266 & 4 & 379 & 2,69 & $\pi / 4$ & 27 & 0,286 & 0,32 & 9,7 & 1,00 \\
\hline 8 & 700 & 4 & 379 & 2,69 & $\pi / 4$ & 27 & 0,286 & 0,32 & 9,7 & 1,00 \\
\hline
\end{tabular}

Деформационное упрочнение плоских образцов двумерных поликристаллов зависит согласно (1) и (4) как от среднего размера зерен $d_{s}$, так и от толщины $D$ деформируемых образцов. Это ясно демонстрируют кривые деформации на рис. 2. Следует также отметить, что эти кривые ограничены степенью деформации 15\%. При больших пластических деформациях необходимо учесть 
особенности формирования ячеистой и фрагментированной дислокационных структур и их вклад в деформационное упрочнение.

\section{ВЫВОДЫ}

В рамках дислокационно-кинетического подхода исследовано пластическое течение плоских образцов двумерных поликристаллов чистых металлов до стадии развитой пластической деформации. На основе имеющихся в литературе данных сформулировано кинетическое уравнение, описывающее эволюцию плотности дислокаций с ростом степени деформации плоского образца двумерного поликристалла с толщиной и средним размером зерен от $~ 50$ мкм и до макроскопических значений в условиях одноосного растяжения с постоянной скоростью деформации при умеренных температурах. Для расчета кривой деформации дислокационно-кинетическое уравнение преобразовано с использованием закона деформационного упрочнения Тейлора и получено аналитическое решение этого уравнения. В качестве примера представлены кривые деформации плоских образцов двумерных поликристаллов чистого алюминия $(99,999$ ат.\%), которые находятся в достаточно хорошем согласии с экспериментальными данными. Предложенная модель позволяет количественно описывать деформационное упрочнение плоских образцов двумерных поликристаллов в зависимости от среднего размера зерен и толщины деформируемых образцов.

\section{БИБЛИОГРАФИЧЕСКИЙ СПИСОК}

1. V.N. Voyevodin, V.V. Bryk, A.S. Kalchenko, I.M. Neklyudov. Simulation technologies in modern radiation material science // Problems of Atomic Science and Technology. Series "Radiation Damage Physics and Radiation Materials Science”. 2014, N 4(92), p. 322.

2. A. Chauhan, D. Litvinov, J. Aktaa. High temperature tensile properties and fracture characteristics of bimodal 12Cr-ODS steel // Journal of Nuclear Materials. 2016, v. 468, p. 1-8.

3. G.A. Vetterick, J. Gruber, P.K. Suri, J.K. Baldwin, M.A. Kirk, P. Baldo, Y.Q. Wang, A. Misra, G.J. Tucker, M.L. Taheri. Achieving Radiation Tolerance through Non-Equilibrium Grain Boundary Structures // Scientific reports. 2017, v. 7(1), p. 12275 .

4. Е.Е. Бадиян, А.Г. Тонкопряд, О.В. Шеховцов, Т.Р. Зетова, Р.В. Шуринов, С.В. Талах, А.В. Дергачова. Особенности структуры двумерных поликристаллов меди, полученных методом рекристаллизации, и характер ее изменения в процессе пластического деформирования // Bonpocbl атомной науки и техники. Серия «Вакуум, чистые материаль, сверхпроводники». 2016, №1(101), с. 8891.

5. E.E. Badiyan, A.G. Tonkopryad, O.V. Shekhovtsov, R.V. Shurinov. Effects of temperature on the laws of plastic deformation and mechanical characteristics foils Al coated with titanium nitride // Bonpocbl атомной науки и техники. Серия «Физика радиационных технологий $и$ радиационное материаловедение». 2016, №2(102), с. 92-98.

6. S. Miyazaki, K. Shibata, H. Fujita. Effect of specimen thickness on mechanical properties of polycrystalline aggregates with various grain sizes // Acta Metall. 1979, v. 27, p. 855-862.

7. P.J. Janssen, T.H. de Keijser, M.G. Geers. An experimental assessment of grain size effects in the uniaxial straining of thin $\mathrm{Al}$ sheet with a few grains across the thickness // Mater. Sci. Eng. 2006, v. A 419, p. 238-248.

8. U.F. Kocks, H. Mecking. Physics and phenomenology of strain hardening: the FCC case // Progr. Mater. Sci. 2003, v. 48, p. 171-273.

9. О.А. Кайбышев, Р.З. Валиев. Границь зерен и свойства металлов. М.: «Металлургия», 1987, 214 с.

10. Г.А. Малыгин. Уравнение эволюции плотности дислокаций и первая стадия деформационного упрочнения кристаллов // ФTT. 1993, т. 35, №5, с. 1328-1342.

11. Г.А. Малыгин. Деформационное упрочнение кристаллов. Размерный, ориентационный и поверхностный эффекты // ФТT. 1993, т. 35, №6, c. $1698-1709$.

12. Г.А. Малыгин. Пластичность и прочность микро- и нанокристаллических материалов // ФTT. 2007, т. 49, №6, с. 961-982.

13. Г.А. Малыгин. Размерные эффекты при пластической деформации микро- и нанокристаллов // ФTT. 2010, т. 52, №1, с. 48-55.

14. Г.А. Малыгин. Влияние поперечного размера образцов с микро- и нанозеренной структурой на предел текучести и напряжение течения // ФTT. 2012, т. 54, №3, с. 523-530.

15. G.I. Taylor. The mechanism of plastic deformation of crystals. Part I. Theoretical // Proc. Roy. Soc. A. 1934, v. 145, N 855, p. 362-387.

16. I.C. Noyan, J.B. Cohen. Residual stress: measurement by diffraction and interpretation. New York: "Springer-Verlag", 1987, p. 44-45.

17. Г.А. Малыгин. Аннигиляция винтовых дислокаций поперечным скольжением как механизм динамического отдыха // ФТТ. 1992, т. 34, №9, c. $2882-2892$. 


\title{
ДИСЛОКАЦЙНА КІНЕТИКА ПРИ ПЛАСТИЧНІЙ ДЕФОРМАЦЇ̈ ДВОВИМІРНИХ ПОЛІКРИСТАЛІВ
}

\author{
С.Ю. Бадіян, А.Г. Тонкопряд, С.В. Фтьомов, О.В. Шеховцов
}

Дислокаційно-кінетичний підхід застосовано до дослідження пластичної течії плоских зразків двовимірних полікристалів чистих металів в умовах одноосного розтягу з постійною швидкістю деформації при помірних температурах. Сформульовано дислокаційно-кінетичне рівняння, в якому враховані роль вільної поверхні плоского зразка, яка є джерелом і стоком дислокацій, і зміцнююча дія наскрізних меж зерен у двовимірному полікристалі. Для розрахунку кривої деформації кінетичне рівняння перетворено 3 використанням закону деформаційного зміцнення Тейлора і отримано аналітичне рішення цього рівняння. На прикладі плоских зразків двовимірних полікристалів чистого алюмінію (99,999 ат.\%) показано, що результати розрахунків досить добре узгоджуються з експериментальними даними.

\section{DISLOCATION KINETICS DURING PLASTIC DEFORMATION OF TWO-DIMENSIONAL POLYCRYSTALS}

\section{E.E. Badiyan, A.G. Tonkopryad, Ye.V. Ftomov, O.V. Shekhovtsov}

The dislocation-kinetic approach is applied to the study of plastic flow of plate specimens of two-dimensional polycrystals of high purity metals under uniaxial tension with a constant strain rate at moderate temperatures. A dislocation-kinetic equation is formulated. It takes into account the role of the free surface of a plate specimen, which is the source and sink of dislocations, and the strengthening effect of through grain boundaries in a twodimensional polycrystal. To calculate tensile stress-strain curves, the kinetic equation was transformed using the Taylor strain hardening law and an analytical solution was obtained for this equation. Using the example of plate specimens of two-dimensional polycrystals of high purity aluminium (99.999 at.\%) it was shown that the calculation results are in good agreement with experimental data. 